\title{
A Planet Finder instrument for the VLT
}

J.-L. Beuzit ${ }^{1}$, M. Feldt ${ }^{2}$, D. Mouillet ${ }^{3}$, C. Moutou ${ }^{4}$, K. Dohlen ${ }^{4}$, P. Puget ${ }^{5}$, T. Fusco ${ }^{6}$, P. Baudoz ${ }^{5}$, A. Boccaletti ${ }^{5}$, S. $_{\text {Udry }}{ }^{7}$,

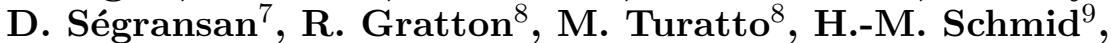

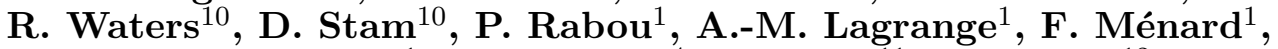
J.-C. Augereau ${ }^{1}$, M. Langlois ${ }^{4}$, F. Vakili ${ }^{11}$, L. Arnold ${ }^{12}$, T. Henning ${ }^{2}$, D. Rouan $^{5}$, M. Kasper ${ }^{13}$ and N. Hubin ${ }^{13}$

${ }^{1}$ Laboratoire d'Astrophysique de Grenoble, BP 53, F-38041 Grenoble Cedex 9, France email: Jean-Luc.Beuzit@obs.ujf-grenoble.fr

${ }^{2}$ Max-Planck-Institut für Astronomie, Königstuhl 17, D-69117 Heidelberg, Germany

${ }^{3}$ Laboratoire d'Astrophysique de Toulouse et Tarbes, BP 826, F-65008 Tarbes Cedex, France

${ }^{4}$ Laboratoire d'Astrophysique de Marseille, BP 8, F-13376 Marseille Cedex, France

${ }^{5}$ Laboratoire d'Etudes Spatiales et d'Instrumentation Astrophysique, F-92190 Meudon, France ${ }^{6}$ ONERA, BP 72, F-92322 Chatillon Cedex, France

${ }^{7}$ Observatoire de Genève, $51 \mathrm{ch}$. Des Maillettes, CH-1290 Sauverny, Switzerland

${ }^{8}$ Osservatorio Astronomico di Padova, Vicolo dell'Osservatorio 5, I-35122 Padova, Italy

${ }^{9}$ Institut für Astronomie, ETH Zentrum, CH-8092 Zurich, Switzerland

${ }^{10}$ Universiteit van Amsterdam, Kruislaan 403, NL-1098 Amsterdam, The Netherlands

${ }^{11}$ Laboratoire Universitaire d'Astrophysique de Nice, Parc Valrose, F-06108 Nice, France

${ }^{12}$ Observatoire de Haute-Provence, F-04870 Saint-Michel-l'Observatoire, France

${ }^{13}$ European Southern Observatory, Karl-Schwarzschild-Strasse 2, D-85748 Garching, Germany

Abstract. Direct detection and spectral characterization of extrasolar planets is one of the most exciting but also one of the most challenging area in modern astronomy. For its second generation instrumentation on the VLT, ESO has supported two phase A studies for a socalled "Planet Finder" dedicated instrument. Based on the results of these two studies, a unique instrument is now considered for first light in early 2010, including a powerful extreme adaptive optics system, various coronagraphs, an infrared differential imaging camera, an infrared integral field spectrograph and a visible differential polarimeter. We will briefly summarize the science objectives and requirements, describe the proposed conceptual design and discuss the main limitations and corresponding instrumental issues of such a system. We will also derive the expected performance of the proposed Planet Finder and present the project organization.

Keywords. instrumentation: adaptive optics, techniques: high angular resolution, stars: lowmass, brown dwarfs, (stars:) planetary systems: formation

\section{Introduction}

The prime objective of a "Planet Finder" instrument for the European Southern Observatory (ESO) Very Large Telescope (VLT) will be the discovery and study of new extra-solar giant planets orbiting nearby stars by direct imaging of their circumstellar environment. The challenge consists in the very large contrast between the host star and the planet, larger than 12.5 magnitudes (or $10^{5}$ in flux ratio), at very small angular separations, typically inside the seeing halo. Therefore the whole design of such an instrument shall be optimized towards reaching the highest contrast in a limited field of view and 
at shortest distances from the central star (Mouillet, et al. 2001). Keeping this prime objective in mind, it is obvious that many other research fields will also benefit from the instrument performance, in the domains of brown dwarfs, proto-planetary disks, mass loss of stars and more marginally for Solar System and extragalactic studies.

This VLT Planet Finder instrument will greatly contribute to the next 10 years of studies in the field of extrasolar planets, already very active, particularly by offering direct detections of planets more massive than Jupiter at various stages of their evolution, in the key separation regime 1 to 100 AUs. Migration mechanisms will then be better understood. The complementarities of direct imaging with other detection methods such as radial velocities and photometric transits, in terms of targets, detection biases and measured planetary parameters, and more specifically the combination with results from other projects like HARPS, COROT, VLTI/PRIMA, NGST and Kepler, will offer promising avenues. The present indications that massive distant planets could be numerous will be firmly confirmed or denied by the VLT Planet Finder, if the number of observed targets with relevant detection limits is statistically acceptable, i.e. greater than typically 150-200. This would in particular fully justify a large effort in an extended observational survey of several hundred nights concentrating on the following classes of targets:

- Nearby young sssociations: they will offer the best chance of detecting low mass planets, since they will have brighter sub-stellar companions, and therefore the greatest number of planets per star observed.

- Stars with known planets: especially any that exhibit long term residuals in their radial velocity curves indicating the possible presence of a more distant planet.

- Nearest stars: measuring these targets will probe the smallest orbits and will thus the only opportunities for detecting planets by directly reflected light.

- Stars aged from 100 Myr to 1 Gyr: planets will still be over-luminous as compared to Solar System planets, so mass limit will be lower than for old systems.

The science cases and potential targets of the VLT Planet Finder are discussed in greater details by Moutou, et al. (2006) in these proceedings.

\section{Science requirements}

The key scientific requirements deriving from the science analysis and driving the design of the instrument are summarized below:

- High contrast to reach giant planets 14 to 16 magnitudes fainter than the host star.

- Access to very small angular separations, 0.1" to 3" from the host star.

- Sensitivity and optimal performance for targets up to visible magnitude $\sim 10$, for building a potential target list in which the sample volume is consistent with the objectives (more than 500 targets in total, with 200 high priority targets).

- Sensitivity to faint companions down to magnitude $\mathrm{H} \sim 24$.

- Access to an extended spectral domain at low resolution, for the characterization of the detected objects, at a resolving power $\sim 30$.

- Sensitivity to extended sources down to $\sim 17$ (respectively 20) magnitudes/"2 at less than $0.2 "$ (respectively $3 "$ ) from the host star. 


\section{Instrument concept}

To fullfil these science requirements, the proposed instrument will include the following main features:

- A common path optical bench, transmitting the telescope beam via the various correcting elements of the adaptive optics system (pupil tip-tilt and pupil de-rotator, deformable mirror, image tip-tilt mirror, atmospheric dispersion compensator...) and via the coronagraphic unit (apodizer, coronagraphic mask, Lyot stop) to the adaptive optics wavefront sensor and to the science channels. A conceptual design of the Planet Finder instrument is presented in Figure 1.

- An eXtreme AO (XAO) system which will fulfill the following three high level requirements: 1) ensure the measurement and correction of the turbulent phase perturbations, of the telescope and system common optics aberrations and of the non-common path aberrations (main AO loop); 2) ensure an extremely high stability of the optical axis at the level of the coronagraphic mask; 3) ensure the measurement and the correction of any pupil motion. A global trade-off combining error budget analysis, optical design, technological aspects, cost and risk issues has led to the following XAO system main characteristics: a $41 \times 41$ actuator deformable mirror, a very fast $(>1 \mathrm{kHz})$ and accurate (0.5 mas) tip-tilt mirror, a spatially filtered Shack-Hartmann wavefront sensor working in the visible at high frequency $(>1 \mathrm{kHz})$, a mixed control law with a Kalman filter law for the tip-tilt control and an optimal modal gain integrator law for the deformable mirror control. In addition an off-line calibration of the non-common path aberrations is foreseen. These aberrations will be compensated in closed loop by a modification of the wavefront sensor references. The expected Strehl ratio will be $90 \%$ in $\mathrm{H}$ band under reasonably good seeing conditions. This XAO system is described in more details by Fusco, et al. (2006) in these proceedings.

- A coronagraphic unit, including a classical Lyot device as well as one or two phase mask devices, allowing to reach very small separations between the host star and the planet candidates. The final choice of these phase mask devices will depend on the results of on-going research and development activities but they are likely to include a 4-Q and an apodized Lyot coronagraph. See for instance Abe, et al. (2006), Soummer, Ferrari \& Aime (2006) and Mawet \& Riaud (2006) in these proceedings for a description of some of these coronagraphic devices.

- An Infra-Red Dual Imaging Spectrograph (IRDIS), the main science module, dedicated to wide-field infrared imaging in one or two simultaneous spectral bands or two orthogonal polarizations as well as low resolution long slit spectroscopy. This main observing mode of IRDIS is based on the principle of differential imaging initially proposed by Racine, et al. (1999) and recently demonstrated on the VLT NACO instrument with the SDI device (Biller, et al. 2006; Close, et al. 2006).

- An Integral Field Spectrograph (IFS), working from $0.95 \mu \mathrm{m}$ to $1.7 \mu \mathrm{m}$ and providing low spectral resolution $(\mathrm{R} \sim 30)$ over a limited, $3 " \times 3$ ", field of view (Berton, et al. 2006).

- A visible dual imaging polarimeter (ZIMPOL), working between $0.65 \mu \mathrm{m}$ and $0.95 \mu \mathrm{m}$ (Schmid, et al. 2006). Due to its innovative lock-in technique, it can achieve polarimetric precisions better than $10^{-5}$ on a localized signal measured differentially against a smooth background.

For stability reasons, the instrument will not be directly attached to the telescope Nasmyth rotator but rather installed on the Nasmyth platform. All the above described 


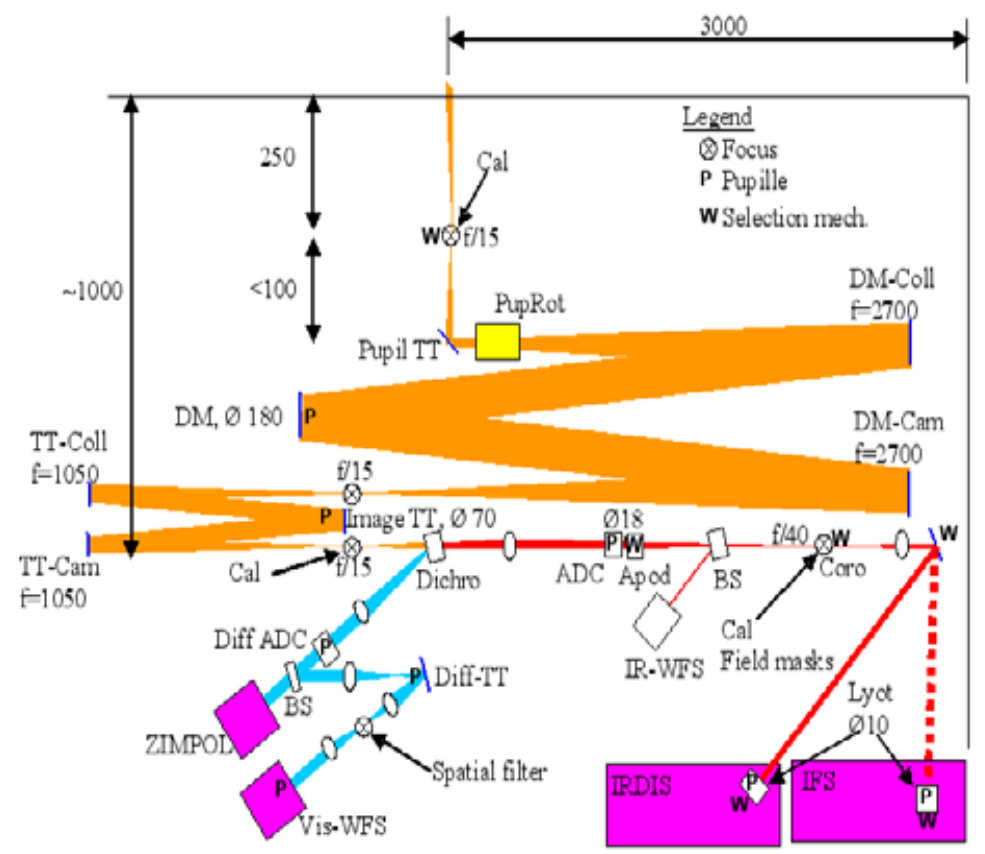

Figure 1. Conceptual design of the VLT Planet Finder instrument.

modules will be located on a fixed bench, covered for protection against dust contamination and controlled for flexures, vibrations and temperature variations.

\section{Expected performances}

End-to-end simulations of the instrument show that contrasts between $10^{5}$ and $10^{6}$ can be reached depending of the characteristics of the host stars, the foreseen separation and the observing mode. Several papers (Boccaletti, et al. 2006; Schmid, et al. 2006 and Berton, et al. 2006) in these proceedings describe the end-to-end models and discuss the expected performance of the VLT Planet Finder instrument for the various focal modes in more details.

An example of the achieved constrast is given as an illustration in Figure 2, for the dual imaging mode.

\section{Project organization}

Following two concurrent phase A studies, the ESO Scientific and Technical Committee has recommended in April 2005 to investigate the feasibility and interest of taking benefit from both studies by proposing an optimized instrument including the XAO system, coronagraphic devices and differential imaging camera proposed by one consortium as well as the Integral Field Spectrograph and ZIMPOL visible dual imaging polarimeter proposed by the other consortium. The on-going post-phase A study shall validate the interest of such an approach in terms of scientific return and its feasibility in terms of system analysis and project management. 


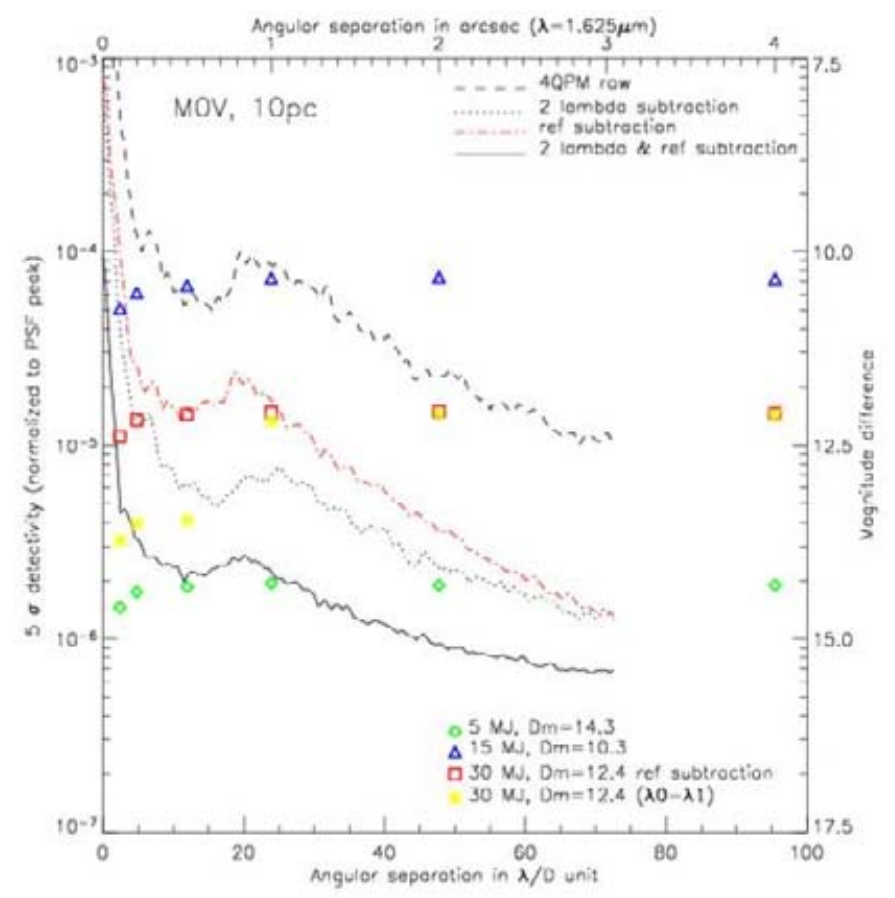

Figure 2. Typical contrast achieved by the proposed VLT Planet Finder instrument in the case of a 1 Gyr M0 star at $10 \mathrm{pc}$, observed in median seeing conditions using the dual imaging mode.

The current development plan, based on a kick-off date beginning of 2006, foresees a first light for the VLT Planet Finder by the spring of 2010. The consortium includes several European institutes, namely: Laboratoire d'Astrophysique in Grenoble (P.I. institute), Max-Planck Institüt für Astronomie in Heidelberg (co-P.I. institute), Laboratoire d'Astrophysique in Marseille, Laboratoire d'Etudes Spatiales et d'Instrumentation en Astrophysique de l'Observatoire de Paris, ONERA, Observatoire de Genève, Osservatorio Astronomico di Padova, Institute of Astronomy of the Zurich College of Technology, Astronomical Institute of the University of Amsterdam, ASTRON and ESO, with contributions from Laboratoire Universitaire d'Astrophysique in Nice, Observatoire de Haute-Provence and Osservatorio Astronomico di Capodimonte in Napoli. The total cost of the instrument is estimated around 9 MEuros.

\section{Conclusion}

The considered Planet Finder instrument, optimized for very high contrast imaging around an extensive sample of stars, and its operation model including in particular a large survey strongly supported by the building consortium will allow the direct detection of a sample of giant planets in a variety of conditions. Such a return in the proposed schedule (first light in 2010) will provide a timely and critical contribution to the highly competitive research field of extra solar planets: formation, evolution and characterization. This observational approach will provide specific information, complementary to other observational techniques (radial velocities, photometric transits, thermal IR imaging, etc.) and absolutely necessary to prepare for the foreseen next generation challenges, from the ground with Extremely Large Telescopes of from space with coronagraphic imaging telescopes (TPF-C) and interferometric instruments (DARWIN). 


\section{References}

Abe, L., Tamura, M., Nakagawa, T., Enya, K., Tanaka, S., Fujita, K., Nishikawa, J., \& Murakami, N. 2006, these proceedings

Berton, A., Feldt, M., Gratton, R., Henning, T., Hippler, S., \& Turatto, M. 2006, these proceedings

Biller, B., Close, L., Lenzen, R., Brandner, W., McCarthy, D., Masciadri, E., Nielsen, E., \& Hartnung, M. 2006, these proceedings

Boccaletti, A., Mouillet, D., Fusco, T., Baudoz, P., Cavarroc, C., Beuzit, J.-L., Moutou, C., \& Dohlen, K. 2006, these proceedings

Close, L., Guirado, J., Nielsen, E., Biller, B., Brandner, W., Lenzen, R., Hartnung, M., Lidman, C., \& Mamajek, E. 2006, these proceedings

Fusco, T., Rousset, G., Beuzit, J.-L., Mouillet, D., \& Dohlen, K. 2006, these proceedings

Mawet, D. \& Riaud, P. 2006, these proceedings

Mouillet, D., Beuzit, J.-L., Chauvin, G., \& Lagrange, A-M. 2001, in J. Bergeron \& G. Monnet (eds), Scientific Drivers for ESO Future VLT/VLTI Instrumentation, ESO Astrophysics Symposia, p. 258

Moutou, C., Beuzit, J.-L., Gratton, R., Mouillet, D., Allard, F., Augereau, J.-C., Boccaletti, A., Desidera, S., Feldt, M., Henning, T., Lagrange, A.-M., Longmore, A., Ménard, F., Schmid, H.M., Stam, D., Udry, S., \& Waters, R. 2006, these proceedings

Racine, R., Walker, G., Nadeau, D., Doyon, R., \& Marois, C. 1999, PASP 111, 587

Schmid, H.M., Beuzit, J.-L., Feldt, M., Gissler D., Gratton, R., Henning, T., Joos, F., Kasper, M., Lenzen, R., Mouillet, D., Moutou, C., Quirrenbach, A., Stam, D., Thalmann, C., Tinbergen, J., Vérinaud, C., Waters, R., \& Wolstencroft, R. 2006, these proceedings

Soummer, R., Ferrari, A., \& Aime, C. 2006, these proceedings 